\title{
Positive Real Synthesis Using Matrix Inequalities for Mechanical Networks: Application to Vehicle Suspension
}

\author{
Christakis Papageorgiou and Malcolm C. Smith, Fellow, IEEE
}

\begin{abstract}
This paper presents a procedure for the synthesis of positive real controllers based on matrix inequalities. Problems with $\mathcal{H}_{2}$ and $\mathcal{H}_{\infty}$ cost are considered and the resulting bilinear matrix inequality problems are solved using local, iterative algorithms. The procedure is applied to the synthesis of passive suspensions for the optimization of certain performance measures for a quarter-car model. The characterization of the positive real constraint using matrix inequalities and the use of a new mechanical element called the inerter, permits the optimization over the entire class of positive real admittances and the realization of the resulting admittance using passive elements. The optimization results are compared with previous results obtained using optimization over fixed-structure admittances. The proposed method can reproduce the previous results and achieve better results in certain cases. Results of the experimental testing of a mechanical network involving an inerter are presented.
\end{abstract}

Index Terms - Inerter, linear matrix inequalities, mechanical device, optimization over positive real admittances, synthesis of passive mechanical networks, vehicle suspensions.

\section{INTRODUCTION}

$\mathbf{P}$ OSITIVE real systems occur in many applications, for example, mechanical structures with collocated sensors and actuators, passive electrical networks (those with only resistors, inductors, and capacitors), and passive mechanical networks (those with masses, dampers, and springs). Positive real systems have motivated the design of strictly positive real compensators since the negative feedback interconnection of a positive real plant with a strictly positive real compensator is asymptotically stable.

Recently, a new mechanical network element termed the "inerter" was introduced as an alternative to the mass element for synthesis of mechanical networks [1]. In the context of vehicle suspensions this was exploited in [2] by optimizing standard performance measures over low-order fixed-structure admittances. The present paper considers the more general class of positive-real functions and seeks to use matrix inequalities as a tool for optimization.

Manuscript received January 31, 2005; revised September 6, 2005. Manuscript received in final form November 29, 2005. Recommended by Associate Editor C.-Y. Su. This work was supported in part by the Engineering and Physical Sciences Research Council, U.K.

C. Papageorgiou is with the Department of Electrical and Computer Engineering, University of Cyprus, Nicosia 1678, Cyprus (e-mail: cpapageo@ ucy.ac.cy).

M. C. Smith is with the Control Group, Engineering Department, Cambridge University, Cambridge CB2 1PZ, U.K. (e-mail: mcs@eng.cam.ac.uk).

Digital Object Identifier 10.1109/TCST.2005.863663
Linear matrix inequalities (LMIs) [3] have emerged as a powerful paradigm and design technique for a variety of linear control problems such as $\mathcal{H}_{2}$ and $\mathcal{H}_{\infty}$ synthesis. Since solving an LMI is a convex optimization problem, such formulations can be solved efficiently using interior-point algorithms. LMIs have also been successful in formulating and solving multiobjective control problems in which various performance specifications (both in the frequency and the time domain) are used for various input-output channels. It was shown in [4] that using a multiobjective formulation, the ride comfort and suspension travel could be improved for a vehicle suspension system.

The problem of synthesizing positive real compensators can be formulated using matrix inequalities since the positive real property of a system can be expressed as an LMI using the positive real lemma [3]. Considerable research has been conducted toward the synthesis of positive real controllers that achieve a level of $\mathcal{H}_{2}$ performance for the control of flexible structures. In [5], a suboptimal version of this problem is shown to be a convex optimization problem and expressed in the form of an LMI. Both the $\mathcal{H}_{2}$ and the positive real constraint are characterized using a common Lyapunov function. In [6], an iterative LMI procedure is proposed for the same problem with the difference that two separate Lyapunov functions are considered for the $\mathcal{H}_{2}$ performance and the positive real constraint. Both methods require that the order of the controller is the same as the order of the generalized plant.

In this paper, the $\mathcal{H}_{2}$ and $\mathcal{H}_{\infty}$ positive real synthesis problems are formulated as bilinear matrix inequality (BMI) problems. In each case an optimization approach is used to find locally optimal solutions. The proposed algorithms allow for any order controller to be considered which is important when searching for a simple network realization of a given positive real function. It will be demonstrated using the quarter-car model that the matrix inequality approach can reproduce the results and give improvements over the previous optimization method.

\section{Synthesis OF ONE-PORT MECHANICAL NETWORKS}

A mechanical network of pure translational type consists of mechanical elements (such as springs, masses, dampers, and levers), which are interconnected in a rigid manner. The pair of end-points of the spring and damper are called nodes or terminals. In a mechanical system, a port is a pair of nodes to which an equal and opposite force $F$ is applied and which experience a relative velocity $v$. The force, which is a through variable, involves a single measurement point and requires the system to 
be severed at that point to make the measurement. The velocity, which is an across variable, can be measured without breaking into the system and the relevant quantity is the difference of the variable between the two points. The concept of through and across variables is natural also for electrical networks and motivates the force-current analogy [7], which is power preserving, with the following correspondences:

$$
\begin{aligned}
\text { force } & \leftrightarrow \text { current } \\
\text { velocity } & \leftrightarrow \text { voltage } \\
\text { mechanical ground } & \leftrightarrow \text { electrical ground } \\
\text { kinetic energy } & \leftrightarrow \text { electrical energy } \\
\text { potential energy } & \leftrightarrow \text { magnetic energy }
\end{aligned}
$$

The impedance $Z(s)$ of a network is defined as the ratio of the across variable to the through variable and the admittance $Y(s)$ is defined as the reciprocal of the impedance. Thus, mechanical admittance is the ratio of force to velocity.

\section{A. Positive Real Functions}

A mechanical one-port network with force-velocity pair $(F, v)$ is defined to be passive if for all admissible $v, F$ which are square integrable on $(-\infty, T]$

$$
\int_{-\infty}^{T} F(t) v(t) d t \geq 0
$$

By definition, a passive network can not deliver energy to the environment. As shown in [8] and [9], the network is passive if and only if the following condition is satisfied: $Z(s)$ is analytic in $\operatorname{Re}(s)>0, Z(j \omega)+Z(j \omega)^{*} \geq 0$, for all $\omega$ at which $Z(j \omega)$ is finite, and any poles of $Z(s)$ on the imaginary axis or at infinity are simple and have a positive residue. Any real-rational function $Z(s)$ satisfying the above is called positive real. The same condition holds for admittances $Y(s)$.

The following fundamental theorem is used in electrical circuit synthesis of positive real impedances and admittances.

Theorem 2.1 [9], [10]: Consider any real-rational function $Z(s),(Y(s))$ which is positive real. There exists a one-port electrical network whose impedance (admittance) equals $Z(s)(Y(s))$ which consists of a finite interconnection of inductors, resistors, and capacitors.

\section{B. The Inerter}

It was pointed out in [1] that Theorem 2.1 cannot be applied directly to the synthesis of mechanical networks because of the fact that the mass element is analogous to a grounded capacitor. Thus, the mechanical analog of an electrical network with ungrounded capacitors can not be realized with the use of springs,

\begin{tabular}{|c|c|c|c|}
\hline \multicolumn{2}{|c|}{ Mechanical } & \multicolumn{2}{|c|}{ Electrical } \\
\hline $\begin{array}{l}\overrightarrow{v_{2}} \\
\frac{d F}{d t}=k\left(v_{2}-v_{1}\right)\end{array}$ & $\begin{array}{r}Y(s)=\frac{k}{s} \\
\text { spring }\end{array}$ & $\frac{d i}{d t}=\frac{1}{L}\left(v_{2}-v_{1}\right)$ & $\begin{array}{l}Y(s)=\frac{1}{L s} \\
\text { inductor }\end{array}$ \\
\hline $\begin{array}{l}\overrightarrow{v_{2}} \\
F=b \frac{d\left(v_{2}-v_{1}\right)}{d t}\end{array}$ & $\begin{array}{l}Y(s)=b s \\
\text { inerter }\end{array}$ & $\begin{array}{l}\stackrel{i}{v_{2}}|| \dot{v}_{1}^{i}- \\
\quad i=C \frac{d\left(v_{2}-v_{1}\right)}{d t}\end{array}$ & $\begin{array}{l}Y(s)=C s \\
\text { capacitor }\end{array}$ \\
\hline 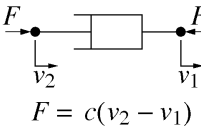 & $\begin{array}{l}Y(s)=c \\
\text { damper }\end{array}$ & $\begin{array}{l}\stackrel{\bullet}{v_{2}} \longrightarrow \stackrel{i}{\stackrel{i}{\rightarrow}} \\
i=\frac{1}{R}\left(v_{2}-v_{1}\right)\end{array}$ & $\begin{array}{l}Y(s)=\frac{1}{R} \\
\text { resistor }\end{array}$ \\
\hline
\end{tabular}
dampers, and masses. This imposes a restriction on the class of passive mechanical impedances which can be physically realized. This restriction is lifted by defining a new mechanical element, the inerter, which is the mechanical analog of the ungrounded capacitor.
Fig. 1. Circuit symbols and correspondences with defining equations and admittance $Y(s)$

Definition 2.1 (Inerter [1]): The (ideal) inerter is a mechanical two-terminal device with the property that the equal and opposite force applied at the nodes is proportional to the relative acceleration between the nodes, i.e., $F=b\left(\dot{v}_{2}-\dot{v}_{1}\right)$ where $v_{1}, v_{2}$ are the velocities of the two terminals and $b$ is a constant of proportionality called the inertance, which has units of kilograms.

The element correspondences in the force-current analogy with the inerter replacing the mass element are shown in Fig. 1.

Prototype inerters have been built at Cambridge University Engineering Department (CUED) using the following: 1) a plunger sliding in a cylinder which drives a flywheel through a rack, pinion and gears [2] and 2) a ball-screw mechanism. Experiments using the latter device are described in Section VIII of the present paper.

\section{The Control Synthesis Paradigm}

In [2], mechanical networks comprising springs, dampers, and inerters were studied for use in passive suspensions for both quarter-car and full-car vehicle models. Performance advantages were found for ride comfort and handling compared to conventional passive suspension struts. The approach in [2] was to consider various fixed-structure admittances which contained at most one inerter and one damper. The parameter values of the components were tuned in order to optimize the various performance measures. This method addresses only a small part of the class of positive real admittances that can be physically realized. In order to be able to synthesize admittances over the whole class of positive real functions, we use a control synthesis paradigm along with a state-space characterization of positive realness. The search for positive real admittances is formulated as a search for positive real "controllers" $K(s)$ as shown in Fig. 2. The characterization of positive realness of the controller is achieved with the following result. This approach requires the order of the controller to be specified in the optimization procedure.

Lemma 2.2 (Positive Real Lemma [3]): Given that

$$
K(s)=\left[\begin{array}{c|c}
A_{k} & B_{k} \\
\hline C_{k} & D_{k}
\end{array}\right]=C_{k}\left(s I-A_{k}\right)^{-1} B_{k}+D_{k}
$$




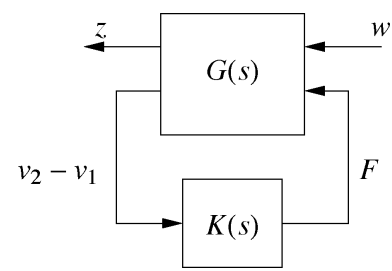

Fig. 2. Control synthesis paradigm applied for the synthesis of a positive real admittance $K(s)$.

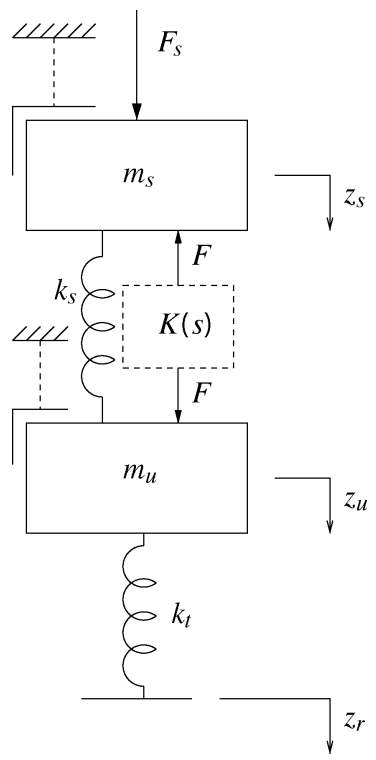

Fig. 3. Quarter-car vehicle model with predetermined static stiffness.

then $K(s)$ is positive real if and only if there exists $P_{k}>0$ that satisfies the LMI

$$
\left[\begin{array}{cc}
A_{k}^{T} P_{k}+P_{k} A_{k} & P_{k} B_{k}-C_{k}^{T} \\
B_{k}^{T} P_{k}-C_{k} & -D_{k}^{T}-D_{k}
\end{array}\right] \leq 0 .
$$

\section{VEHICLE SUSPENSIONS}

\section{A. The Quarter-Car Model}

The quarter-car model presented in Fig. 3 is the simplest model to consider for suspension design. It consists of the sprung mass $m_{s}$, the unsprung mass $m_{u}$, and a tyre with spring stiffness $k_{t}$. The suspension strut provides an equal and opposite force on the sprung and unsprung masses and is assumed to be a passive mechanical admittance $Y(s)=K(s)+\left(k_{s} / s\right)$, where $K(s)$ is positive real and has no pole at $s=0$. In this paper, we fix the parameters of the quarter-car model as $m_{s}=250 \mathrm{~kg}, m_{u}=35 \mathrm{~kg}, k_{t}=150 \mathrm{kN} / \mathrm{m}$.

\section{B. Performance Measures}

There are a number of practical design requirements for a suspension system, such as passenger comfort, handling, tyre normal loads, and limits on suspension travel, which require careful optimization. In the quarter-car model these can be translated approximately into specifications on the transfer functions from the disturbance signals $F_{s}$ and $z_{r}$ to the signals $z_{s}$ and $z_{u}$. The performance measures used in this paper are discussed in detail in [2]. The first two assume a standard rational power spectrum for the road disturbances, while the third relates to the effect of loads on the sprung mass.

For the ride comfort we use the root-mean-square (rms) body vertical acceleration in response to road disturbances, denoted by $J_{1}$, which is equal to

$$
J_{1}=2 \pi(V \kappa)^{\frac{1}{2}}\left\|s T_{\hat{z}_{r} \rightarrow \hat{z}_{s}}\right\|_{2}
$$

where $V$ is the speed of the car, $\kappa$ is the road roughness parameter, $T_{\hat{z}_{r} \rightarrow \hat{z}_{s}}$ denotes the transfer function from the road disturbance $z_{r}$ to the displacement of the sprung mass $z_{s}$, and $\|\cdot\|_{2}$ is the standard $\mathcal{H}_{2}$ norm.

To characterize road holding we use the rms dynamic tyre load in response to road disturbances, denoted by $J_{3}$, which is equal to

$$
J_{3}=2 \pi(V \kappa)^{\frac{1}{2}}\left\|\frac{1}{s} T_{\hat{z}_{r} \rightarrow k_{t}\left(\hat{z}_{u}-\hat{z}_{r}\right)}\right\|_{2} .
$$

Another factor to be considered is the ability of the suspension to withstand external loads on the sprung mass, e.g., those loads induced by braking, accelerating, and cornering. The following measure is used for this purpose:

$$
J_{5}=\left\|T_{\hat{F}_{s} \rightarrow \hat{z}_{s}}\right\|_{\infty}
$$

where $\|\cdot\|_{\infty}$ represents the $\mathcal{H}_{\infty}$-norm. We will attempt to minimize each performance measure on its own over positive real admittances $Y(s)$ of fixed degree and compare the results with those obtained in [2].

\section{The Quarter-Car Model as a Linear Fractional Transformation for the Optimization of $J_{1}$ and $J_{5}$}

Since the performance measures are expressed as either $\mathcal{H}_{2}$ or $\mathcal{H}_{\infty}$ norms of certain transfer functions, it is proposed to formulate the suspension design problem as a standard $\mathcal{H}_{2}$ or $\mathcal{H}_{\infty}$ controller synthesis problem. This requires that the quarter-car model is written as a linear fractional transformation (LFT) with respect to the unknown, positive real admittance $K(s)$. The interconnection used for the derivation of the generalized, quarter-car plant is given in Fig. 3. We require that the static stiffness of the suspension is determined a priori and is given by $k_{s}$. The equations of motion are given by

$$
\begin{aligned}
\ddot{z}_{s} & =\frac{F_{s}}{m_{s}}-\frac{F}{m_{s}}-\frac{k_{s}}{m_{s}} z_{s}+\frac{k_{s}}{m_{s}} z_{u} \\
\ddot{z}_{u} & =\frac{F}{m_{u}}+\frac{k_{s}}{m_{u}} z_{s}-\frac{k_{s}}{m_{u}} z_{u}+\frac{k_{t}}{m_{u}} z_{r}-\frac{k_{t}}{m_{u}} z_{u} \\
\hat{F} & =K(s)\left(s \hat{z}_{s}-s \hat{z}_{u}\right) .
\end{aligned}
$$

The external input of the generalized plant is $w=\left[F_{s}, z_{r}\right]^{T}$, while the performance output is given by $z=\left[\dot{z}_{s}, z_{s}\right]^{T}$. Writing the above equations in state-space form with the state vector given by $x=\left[\dot{z}_{s}, z_{s}, \dot{z}_{u}, z_{u}\right]^{T}$ results in the quarter-car, generalized plant

$$
\dot{x}=A x+B\left[\begin{array}{l}
w \\
F
\end{array}\right], \quad\left[\begin{array}{c}
z \\
\dot{z}_{s}-\dot{z}_{u}
\end{array}\right]=C x
$$


where

$$
\begin{aligned}
A & =\left[\begin{array}{cccc}
0 & -\frac{k_{s}}{m_{s}} & 0 & \frac{k_{s}}{m_{s}} \\
1 & 0 & 0 & 0 \\
0 & \frac{k_{s}}{m_{u}} & 0 & -\frac{k_{s}+k_{t}}{m_{u}} \\
0 & 0 & 1 & 0
\end{array}\right] \\
B & =\left[\begin{array}{ccc}
\frac{1}{m_{s}} & 0 & -\frac{1}{m_{s}} \\
0 & 0 & 0 \\
0 & \frac{k_{t}}{m_{u}} & \frac{1}{m_{u}} \\
0 & 0 & 0
\end{array}\right] \\
C & =\left[\begin{array}{cccc}
1 & 0 & 0 & 0 \\
0 & 1 & 0 & 0 \\
1 & 0 & -1 & 0
\end{array}\right] .
\end{aligned}
$$

Let $B_{1}, B_{2}, B_{3}$ be the respective columns of $B$, and let $C_{1}$, $C_{2}, C_{3}$ be the respective rows of $C$. Since the static stiffness is determined ita priori, the admittance $K(s)$ must have zero static stiffness, hence, it may not contain an integrator. This is in fact ensured by the structure of the generalized plant. The transfer function from $F$ to $\dot{z}_{s}-\dot{z}_{u}$ is given by

$$
T_{F \rightarrow\left(\hat{z}_{s}-\hat{z}_{u}\right)}=-\frac{s\left(\left(m_{u}+m_{s}\right) s^{2}+k_{t}\right)}{m_{u} m_{s} s^{4}+s^{2}\left(m_{u} k_{s}+k_{s} m_{s}+k_{t} m_{s}\right)+k_{t} k_{s}} .
$$

Therefore, a "stabilizing controller" $K(s)$ cannot have an integrator because it will cause an right-half plane (RHP) pole-zero cancellation [11, Sec. 12.1].

\section{OPTIMIZATION OF $J_{1}$}

The generalized plant for the $J_{1}$ optimization is formed by considering $z_{r}$ as the external disturbance and $\dot{z}_{s}$ as the performance output. The objective is to find a positive real $K(s)$ so that $\left\|T_{\hat{z}_{r} \rightarrow s \hat{z}_{s}}\right\|_{2}$ is minimized. From (10), the observable and controllable representation of the $J_{1}$ generalized plant is given by

$$
\begin{aligned}
\dot{x} & =A x+B_{2} z_{r}+B_{3} F \\
\dot{z}_{s} & =C_{1} x, \quad \dot{z}_{s}-\dot{z}_{u}=C_{3} x .
\end{aligned}
$$

Given a controller $K(s)$ of order $n_{k}$, with state-space representation as in (2), let the state-space representation of the closedloop system resulting from the interconnection of the generalized plant and the controller be given by

$$
\left[\begin{array}{c}
\dot{x} \\
\dot{x}_{k} \\
\hline \dot{z}_{s}
\end{array}\right]=\left[\begin{array}{cc}
A_{c l} & B_{c l} \\
C_{c l} & 0
\end{array}\right]\left[\begin{array}{c}
x \\
\frac{x_{k}}{z_{r}}
\end{array}\right] .
$$

Theorem 4.1: There exists a positive real controller $K(s)$ of order $n_{k}$ such that $\left\|T_{\hat{z}_{r} \rightarrow s \hat{z}_{s}}\right\|_{2}<v$ and $A_{c l}$ is stable, if and only if the following problem is feasible for some $X_{c l}>0, X_{k}>0$, $Q, v^{2}$ and $A_{k}, B_{k}, C_{k}, D_{k}$ of compatible dimensions:

$$
\begin{gathered}
{\left[\begin{array}{cc}
A_{c l}^{T} X_{c l}+X_{c l} A_{c l} & X_{c l} B_{c l} \\
B_{c l}^{T} X_{c l} & -I
\end{array}\right]<0, \quad\left[\begin{array}{cc}
X_{c l} & C_{c l}^{T} \\
C_{c l} & Q
\end{array}\right]>0} \\
\operatorname{tr}(Q)<v^{2}, \quad\left[\begin{array}{cc}
A_{k}^{T} X_{k}+X_{k} A_{k} & X_{k} B_{k}-C_{k}^{T} \\
B_{k}^{T} X_{k}-C_{k} & -D_{k}^{T}-D_{k}
\end{array}\right]<0 .
\end{gathered}
$$
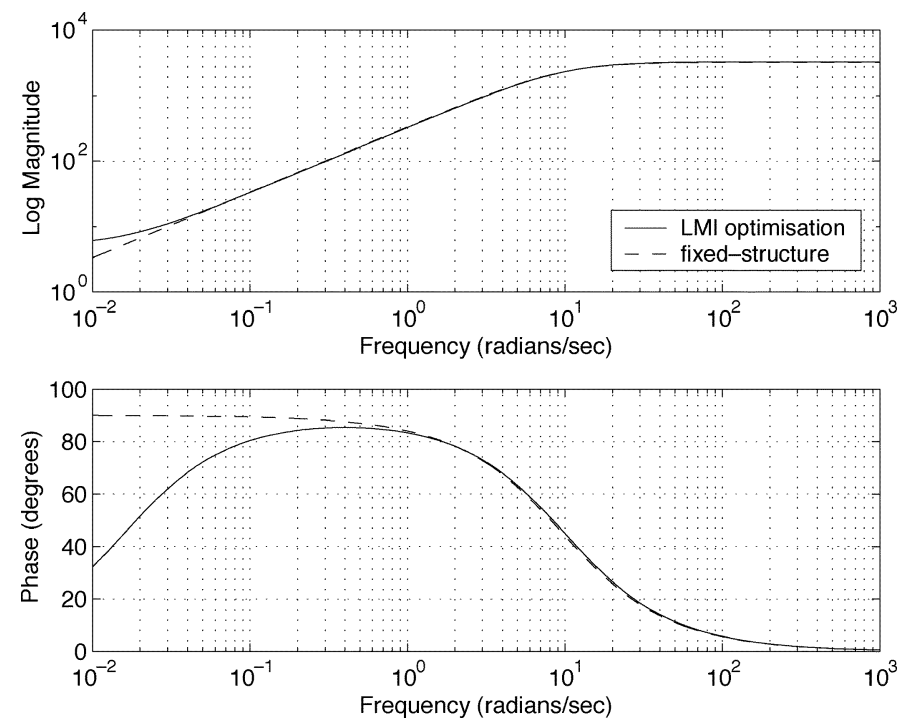

Fig. 4. Comparison of first-order admittances $K(s)$ for the quarter-car model for $k_{s}=60 \mathrm{kN} / \mathrm{m}$.

The first three LMIs are necessary and sufficient conditions for the existence of a stabilizing controller that achieves an upper bound of $v$ on the $\mathcal{H}_{2}$-norm [12]. The fourth LMI further restricts the controller to be positive real. Without the positive real constraint, the $\mathcal{H}_{2}$-synthesis problem can be formulated as an LMI problem as shown in [12]. With the positive real constraint it is not obvious how to do so, hence, an iterative optimization method is employed to solve the BMI problem locally. The method, which is described in [13], is to linearize the BMI using a first-order perturbation approximation, and then iteratively compute a perturbation that "slightly" improves the controller performance by solving an LMI problem. The proposed scheme is already implemented in YALMIP [14], which is a MATLAB toolbox for rapid prototyping of optimization problems. A feasible starting point must be given to the algorithm.

\section{A. $J_{1}$ Optimization Results}

There are two issues to be investigated regarding the proposed synthesis method. The first is whether it can reproduce the results of the fixed-structure optimization and the second, whether it can give improved levels of performance exploiting the optimization over the entire class of positive real admittances.

An example is presented that demonstrates that the proposed method is successful in reproducing the fixed-structure optimization results. The quarter-car model is considered with static stiffness $k_{s}=60 \mathrm{kN} / \mathrm{m}$. The fixed-structure admittance proposed in [2] is a damper in series with an inerter, i.e., $K(s)=$ $c b s /(c+b s)$, which achieves a value of $J_{1}=1.5851$ for $c=$ $3224 \mathrm{Ns} / \mathrm{m}$ and $b=334 \mathrm{~kg}$. The admittance $K(s)$ calculated by YALMIP is given by

$$
K(s)=\frac{3276 s+52}{s+10}=5.2+\left(\frac{1}{3271}+\frac{1}{327.1 s}\right)^{-1}
$$

and achieves $J_{1}=1.5855$. A comparison of the two admittances is shown in Fig. 4. The YALMIP admittance cannot ex- 


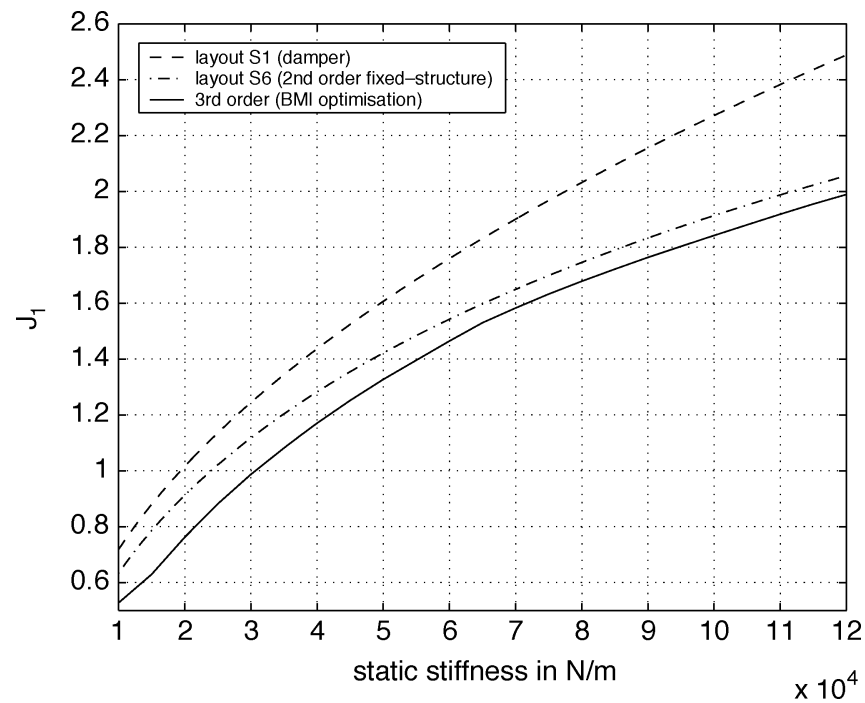

Fig. 5. Improvement in $J_{1}$ when using higher order admittances.

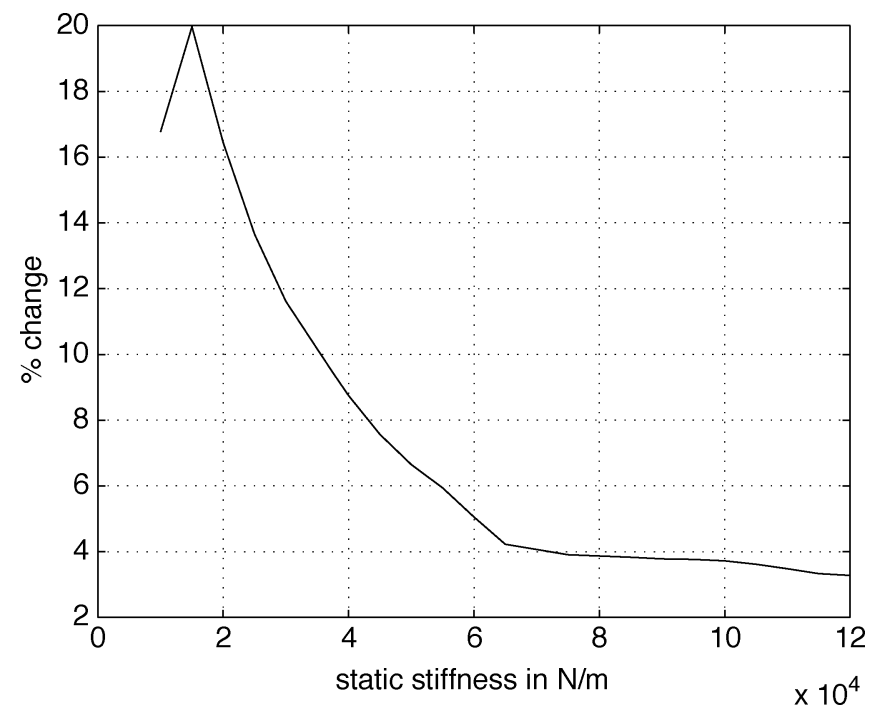

Fig. 6. Percentage improvement in $J_{1}$ when $K(s)$ is third-order.

actly match the fixed-structure admittance because it can only optimize over the class of strictly positive real controllers which implies $\operatorname{Re}(K(j \omega))>0$.

Apart from being able to approximately achieve the fixed-structure admittances suggested in [2] it is useful to know whether $J_{1}$ can be reduced further by optimizing over higher order admittances. The highest order admittance used in [2] was of second order. The best results obtained so far with third-order admittances are presented in Fig. 5 as a function of the static stiffness of the suspension, along with previous results related to fixed-structure admittances. The "best" fixed-structure admittance found in [2] is a parallel connection of a damper and centering spring in series with a parallel connection of an inerter and centering spring and was given the name layout $S 6$. The percentage improvement is calculated with respect to the values of $J_{1}$ achieved by the $S 6$ fixed-structure admittance. The results are shown in Fig. 6. The optimization algorithm was run for values of static stiffness in the range $10-120 \mathrm{kN} / \mathrm{m}$ at a spacing of $5 \mathrm{kN} / \mathrm{m}$.

\section{OPTIMIZATION OF $J_{3}$}

\section{A. Generalized Plant for the Optimization of $J_{3}$}

The performance output corresponding to $J_{3}$ is given by $\int k_{t}\left(z_{u}-z_{r}\right)$. This is implemented by adding an integrator on $k_{t}\left(z_{u}-z_{r}\right)$, thus creating one more state for the generalized plant. The generalized plant for the optimization of $J_{3}$ is given by

$$
\begin{aligned}
& {\left[\begin{array}{c}
\ddot{z}_{s} \\
\dot{z}_{s} \\
\ddot{z}_{u} \\
\dot{z}_{u} \\
\dot{x}_{1}
\end{array}\right]=\left[\begin{array}{ccccc}
0 & -\frac{k_{s}}{m_{s}} & 0 & \frac{k_{s}}{m_{s}} & 0 \\
1 & 0 & 0 & 0 & 0 \\
0 & \frac{k_{s}}{m_{u}} & 0 & -\frac{k_{s}+k_{t}}{m_{u}} & 0 \\
0 & 0 & 1 & 0 & 0 \\
0 & 0 & 0 & k_{t} & 0
\end{array}\right]\left[\begin{array}{c}
\dot{z}_{s} \\
z_{s} \\
\dot{z}_{u} \\
z_{u} \\
x_{1}
\end{array}\right]} \\
& +\left[\begin{array}{cc}
0 & -\frac{1}{m_{s}} \\
0 & 0 \\
\frac{k_{t}}{m_{u}} & \frac{1}{m_{u}} \\
0 & 0 \\
-k_{t} & 0
\end{array}\right]\left[\begin{array}{c}
z_{r} \\
F
\end{array}\right] \\
& {\left[\begin{array}{c}
\int k_{t}\left(z_{u}-z_{r}\right) \\
\dot{z}_{s}-\dot{z}_{u}
\end{array}\right]=\left[\begin{array}{ccccc}
0 & 0 & 0 & 0 & 1 \\
1 & 0 & -1 & 0 & 0
\end{array}\right]\left[\begin{array}{c}
\dot{z}_{s} \\
z_{s} \\
\dot{z}_{u} \\
z_{u} \\
x_{1}
\end{array}\right]} \\
& +\left[\begin{array}{ll}
0 & 0 \\
0 & 0
\end{array}\right]\left[\begin{array}{c}
z_{r} \\
F
\end{array}\right] .
\end{aligned}
$$

The addition of the extra integrator creates problems since the added state is uncontrollable. Consider the similarity transformation given by

$$
\left[\begin{array}{l}
\dot{z}_{s} \\
z_{s} \\
\dot{z}_{u} \\
z_{u} \\
x_{n}
\end{array}\right]=\left[\begin{array}{ccccc}
1 & 0 & 0 & 0 & 0 \\
0 & 1 & 0 & 0 & 0 \\
0 & 0 & 1 & 0 & 0 \\
0 & 0 & 0 & 1 & 0 \\
m_{s} & 0 & m_{u} & 0 & 1
\end{array}\right]\left[\begin{array}{c}
\dot{z}_{s} \\
z_{s} \\
\dot{z}_{u} \\
z_{u} \\
x_{1}
\end{array}\right] .
$$

The transformed system equations are

$$
\begin{aligned}
& \begin{aligned}
{\left[\begin{array}{c}
\ddot{z}_{s} \\
\dot{z}_{s} \\
\ddot{z}_{u} \\
\dot{z}_{u} \\
\dot{x}_{n}
\end{array}\right]=} & {\left[\begin{array}{ccccc}
0 & -\frac{k_{s}}{m_{s}} & 0 & \frac{k_{s}}{m_{s}} & 0 \\
1 & 0 & 0 & 0 & 0 \\
0 & \frac{k_{s}}{m_{u}} & 0 & -\frac{k_{s}+k_{t}}{m_{u}} & 0 \\
0 & 0 & 1 & 0 & 0 \\
0 & 0 & 0 & 0 & 0
\end{array}\right]\left[\begin{array}{c}
\dot{z}_{s} \\
z_{s} \\
\dot{z}_{u} \\
z_{u} \\
x_{n}
\end{array}\right] } \\
& +\left[\begin{array}{cc}
0 & -\frac{1}{m_{s}} \\
0 & 0 \\
\frac{k_{t}}{m_{u}} & \frac{1}{m_{u}} \\
0 & 0 \\
0 & 0
\end{array}\right]\left[\begin{array}{c}
z_{r} \\
F
\end{array}\right]
\end{aligned} \\
& \begin{aligned}
{\left[\begin{array}{c}
\ddot{z}_{s} \\
\dot{z}_{s} \\
\ddot{z}_{u} \\
\dot{z}_{u} \\
\dot{x}_{n}
\end{array}\right]=} & {\left[\begin{array}{ccccc}
0 & -\frac{k_{s}}{m_{s}} & 0 & \frac{k_{s}}{m_{s}} & 0 \\
1 & 0 & 0 & 0 & 0 \\
0 & \frac{k_{s}}{m_{u}} & 0 & -\frac{k_{s}+k_{t}}{m_{u}} & 0 \\
0 & 0 & 1 & 0 & 0 \\
0 & 0 & 0 & 0 & 0
\end{array}\right]\left[\begin{array}{c}
\dot{z}_{s} \\
z_{s} \\
\dot{z}_{u} \\
z_{u} \\
x_{n}
\end{array}\right] } \\
& +\left[\begin{array}{cc}
0 & -\frac{1}{m_{s}} \\
0 & 0 \\
\frac{k_{t}}{m_{u}} & \frac{1}{m_{u}} \\
0 & 0 \\
0 & 0
\end{array}\right]\left[\begin{array}{c}
z_{r} \\
F
\end{array}\right]
\end{aligned} \\
& {\left[\begin{array}{c}
\int k_{t}\left(z_{u}-z_{r}\right) \\
\dot{z}_{s}-\dot{z}_{u}
\end{array}\right]=\left[\begin{array}{ccccc}
-m_{s} & 0 & -m_{u} & 0 & 1 \\
1 & 0 & -1 & 0 & 0
\end{array}\right]\left[\begin{array}{c}
\dot{z}_{s} \\
z_{s} \\
\dot{z}_{u} \\
z_{u} \\
x_{n}
\end{array}\right]} \\
& +\left[\begin{array}{ll}
0 & 0 \\
0 & 0
\end{array}\right]\left[\begin{array}{c}
z_{r} \\
F
\end{array}\right]
\end{aligned}
$$




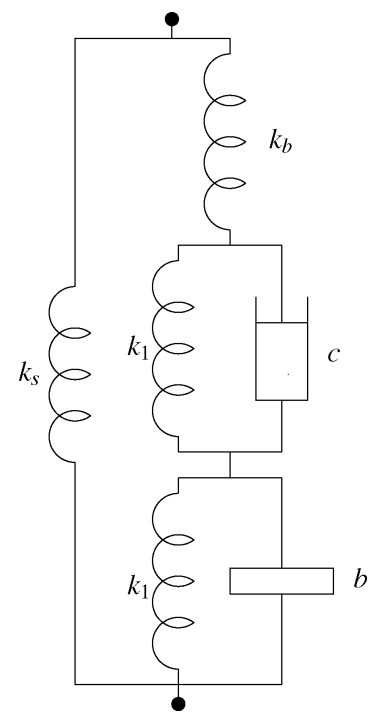

Fig. 7. S7 suspension layout proposed in [2].

It is evident that the state $x_{n}$ is uncontrollable so it can be removed. Furthermore, the output equation

$$
\int k_{t}\left(z_{u}-z_{r}\right)=-m_{s} \dot{z}_{s}-m_{u} \dot{z}_{u}
$$

is divided by $k_{t}$ in order to make the state-space matrices more well-conditioned resulting in

$$
\int\left(z_{u}-z_{r}\right)=-\frac{m_{s}}{k_{t}} \dot{z}_{s}-\frac{m_{u}}{k_{t}} \dot{z}_{u}
$$

The performance measure is now equal to

$$
J_{3}=2 \pi(V \kappa)^{\frac{1}{2}} k_{t}\left\|T_{\hat{z}_{r} \rightarrow \int\left(\hat{z}_{u}-\hat{z}_{r}\right)}\right\|_{2} .
$$

The generalized plant for the $J_{3}$ optimization is finally given by

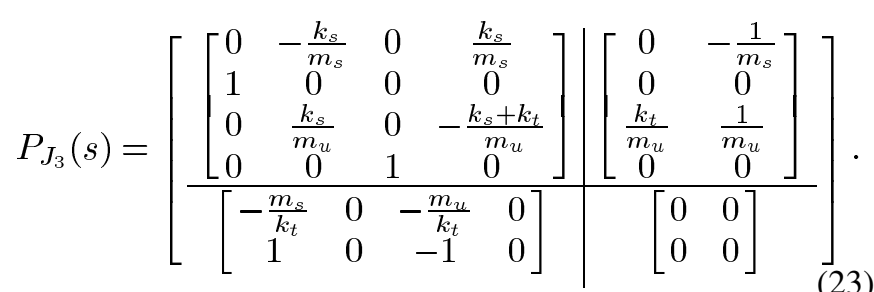

The $J_{3}$ optimization problem is similar to the $J_{1}$ optimization problem, i.e., a positive real controller $K(s)$ is sought to minimize the $\mathcal{H}_{2}$ norm of the closed-loop transfer function. A theorem can be written down which is directly analogous to Theorem 4.1 to give a necessary and sufficient condition for the achievement of a certain performance level. Again, this characterization is in the form of a BMI which can be solved locally using the iterative algorithm implemented in YALMIP.

\section{B. $J_{3}$ Optimization Results}

The optimization of the $J_{3}$ measure was attempted in [2] over fixed structure suspensions. The maximum order of the considered fixed structure admittances was three. The third-order suspension is referred to as $\mathrm{S} 7$ and is shown in Fig. 7. The

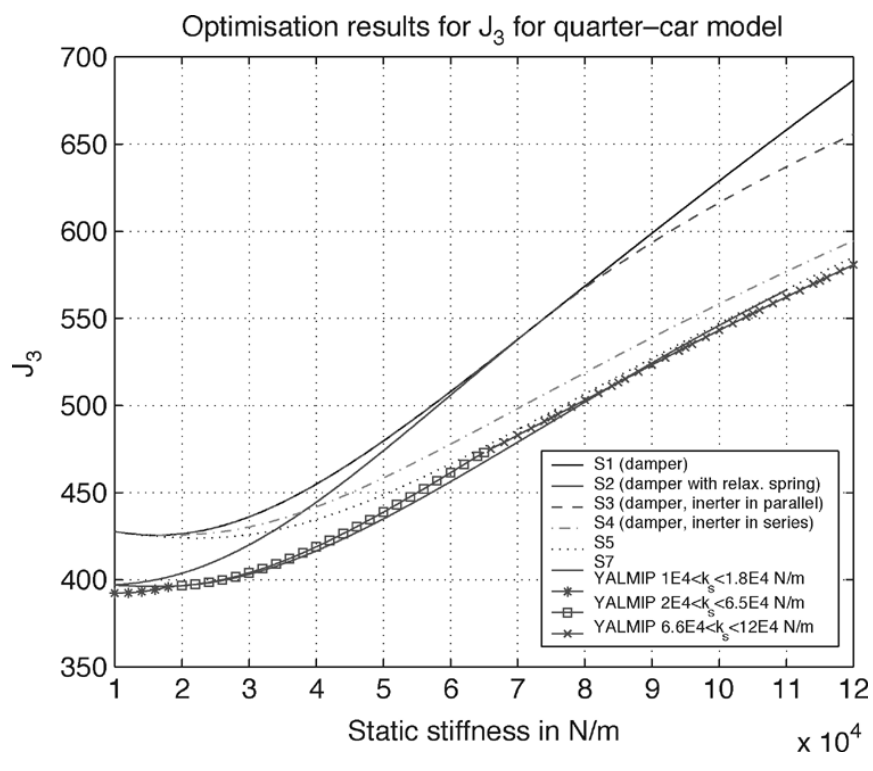

Fig. 8. Comparison of YALMIP optimization results with fixed-structure optimization results for $J_{3}$.

fixed-structure second-order layout is referred to as S5 and is the same as $\mathrm{S} 7$ with the relaxation spring $k_{b}$ removed.

The iterative algorithm implemented in YALMIP was used to optimize $J_{3}$ over general second-order admittances in order to investigate whether $J_{3}$ can be improved further. The optimization was performed for $k_{s}$ ranging from 10 to $120 \mathrm{kN} / \mathrm{m}$ in steps of $2 \mathrm{kN} / \mathrm{m}$. The comparison of the optimization results obtained with YALMIP with those obtained by fixed-structure optimization are presented in Fig. 8. The results exhibit three distinct curves suggesting that the structure of the suspension changes as the static stiffness varies. At low and high stiffness, the YALMIP second-order admittance can do better than both the second-order S5 layout and the third-order S7 layout. An encouraging feature of the optimization algorithm is that it automatically finds the change in the structure of the admittance as the static stiffness varies in order to obtain the minimum value of $J_{3}$. It is of interest to investigate the structure of the suspensions obtained with YALMIP and understand how they differ from the fixed-structure suspensions.

1) The Suspensions Corresponding to the Low Static Stiffness Range $\left(10 \mathrm{kN} / \mathrm{m}<k_{s}<18 \mathrm{kN} / \mathrm{m}\right)$ : As a representative of this class of admittances we consider the admittance of the suspension for $k_{s}=12 \mathrm{kN} / \mathrm{m}$. The admittance is given by

$$
K(s)=\frac{32.566(s+3955)(s+183.43)}{s^{2}+133.71 s+12545}
$$

and it achieves a value of $J_{3}=392.5$. A simpler admittance is constructed by canceling the term $(s+3955)$ and by modifying the term $(s+183.43)$ sufficiently to maintain the positive real character of the new admittance. The new admittance is given by

$$
K_{a p}(s)=\frac{32.566 \times 3955 \times 183.43(s+133)}{s^{2}+133.71 s+12545} .
$$

Its frequency response is compared with the frequency response of $K(s)$ and with the frequency response of the fixed-structure 

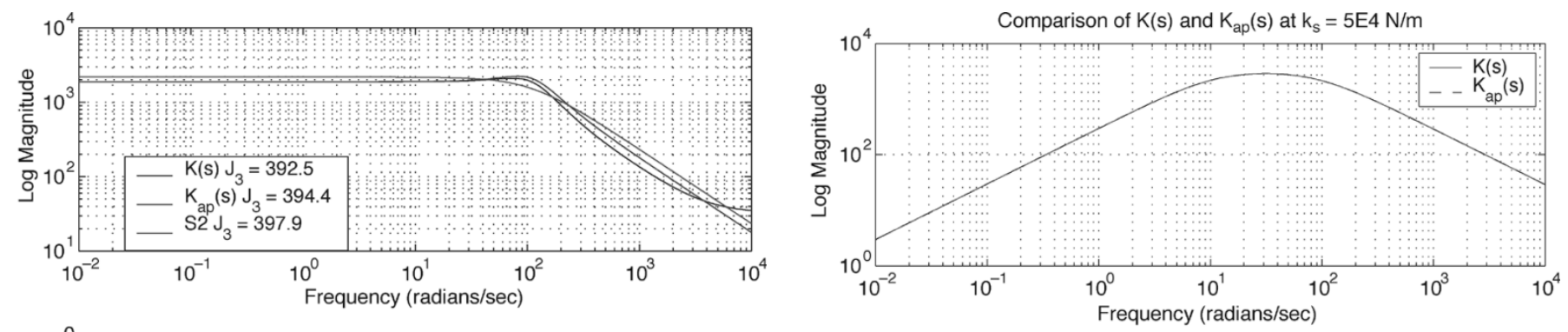

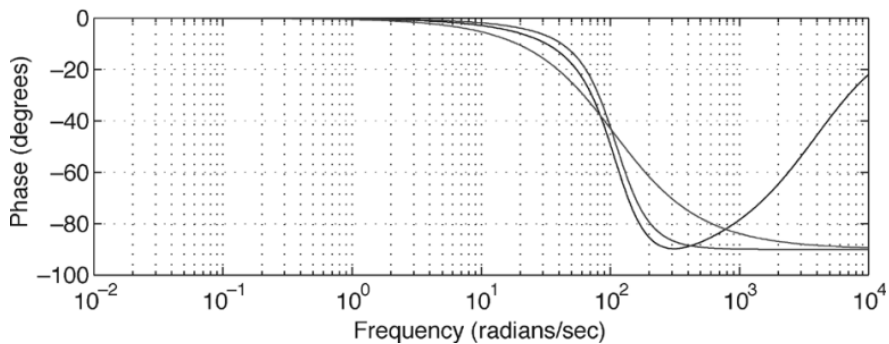

Fig. 9. Comparison of the original and approximate admittances at $k_{s}=$ $12 \mathrm{kN} / \mathrm{m}$

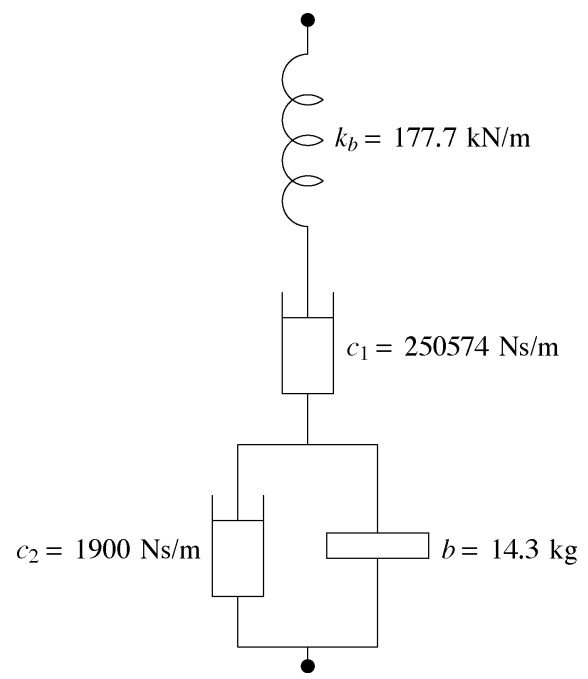

Fig. 10. Suspension layout corresponding to the admittance $K_{a p}(s)$.

admittance S2 (damper in series with relaxation spring, see [2]) in Fig. 9. The approximate admittance achieves $J_{3}=394.4$ and its realization is given in Fig. 10. If we remove the small inertance in the realization of Fig. 10, the suspension layout becomes the same as the S2 layout but with slightly different values for $k_{b}$ and $c$ from the ones obtained in [2]. Therefore, it seems that the $1.5 \%$ improvement in $J_{3}$ does not justify the extra complexity of the realization in Fig. 10 over the S2 layout and the unrealistic damper rate of $c_{1}$ (although a very large damper rate implies a short circuit and, thus, it can be neglected).

2) The Suspensions Corresponding to the Intermediate Static Stiffness Range $\left(20 \mathrm{kN} / \mathrm{m}<k_{s}<65 \mathrm{kN} / \mathrm{m}\right)$ : As a representative of this class of admittances we consider the admittance of the suspension for $k_{s}=50 \mathrm{kN} / \mathrm{m}$. The admittance is given by

$$
K(s)=\frac{1.3(s+222633)\left(s+0.148 \times 10^{3}\right)}{(s+90.3)(s+10.8)}
$$

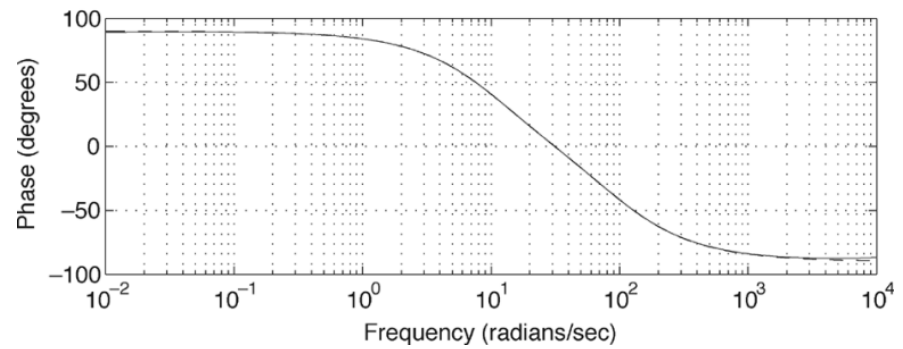

Fig. 11. Comparison of the original and approximate admittances for $k_{s}=$ $50 \mathrm{kN} / \mathrm{m}$.

and it achieves a value of $J_{3}=438.8$. A simpler admittance is constructed by canceling the term $(s+222633)$ and by approximating the term $\left(s+0.148 \times 10^{3}\right)$ with $s$. The approximate admittance is given by

$$
K_{a p}(s)=\frac{288510 s}{(s+90.3)(s+10.8)}
$$

and it achieves $J_{3}=438.8$, i.e., there is practically no degradation in $J_{3}$. The comparison of the frequency responses of the original admittance $K(s)$ with $K_{a p}(s)$ is shown in Fig. 11. A realization of $K_{a p}(s)$ is constructed by noting that,

$$
K_{a p}(s)=\left(\frac{1}{\frac{288510}{s}}+\frac{1}{2857}+\frac{1}{297 s}\right)^{-1} .
$$

It is obvious that $K_{a p}(s)$ is the admittance of the network consisting of an inerter in series with a damper in series with a spring shown in Fig. 12. Note that the layout S7 would be the same as the layout of Fig. 12 if we include centering springs in parallel with the damper and the inerter.

3) The Suspensions Corresponding to the High Static Stiffness Range $\left(66 \mathrm{kN} / \mathrm{m}<k_{s}<120 \mathrm{kN} / \mathrm{m}\right)$ : As a representative of this class of admittances we consider the admittance of the suspension for $k_{s}=90 \mathrm{kN} / \mathrm{m}$. The admittance is given by

$$
K(s)=\frac{3306.1(s+8.08)(s+0.5564)}{s^{2}+12.1 s+152.12}
$$

and achieves $J_{3}=523.3$. A simpler admittance is constructed by making the term $(s+0.5564)$ equal to $s$ and by modifying the remaining terms so that the positive real property is preserved. The approximate admittance is given by

$$
K_{a p}(s)=\frac{3306.1 s(s+13)}{s^{2}+13 s+152.12}
$$




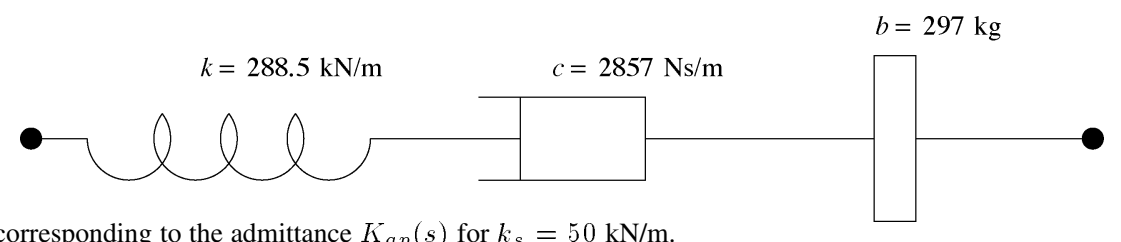

Fig. 12. Suspension layout corresponding to the admittance $K_{a p}(s)$ for $k_{s}=50 \mathrm{kN} / \mathrm{m}$.
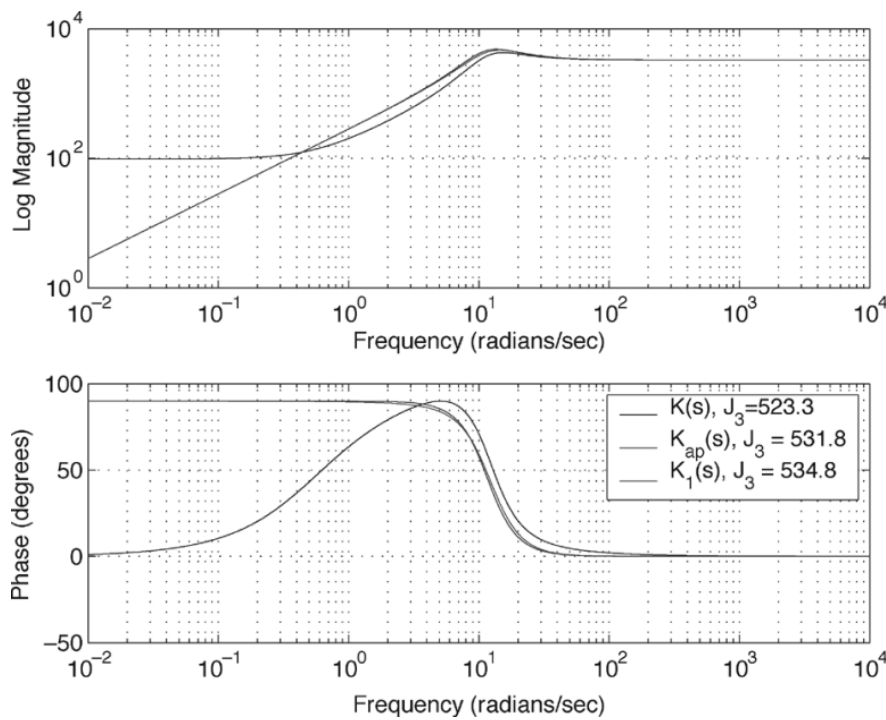

Fig. 13. Comparison of $K(s)$ and $K_{a p}(s)$ at $k s=90 \mathrm{kN} / \mathrm{m}$.

and achieves $J_{3}=531.8$, i.e., there is $1.6 \%$ degradation in $J_{3}$. A comparison of the frequency responses between $K(s)$ and $K_{a p}(s)$ is shown in Fig. 13. A realization for the admittance $K_{a p}(s)$ is found by noting that

$$
\begin{aligned}
& K_{a p}(s) \\
& =3306.1\left(\frac{11.7}{s}+\frac{13 s+16.88}{13 s+169}\right)^{-1} \\
& =3306.1\left(\frac{11.7}{s}+\left(1+\frac{152.12}{13 s+16.88}\right)^{-1}\right)^{-1} \\
& =\left(\frac{1}{282.6 s}+\left(3306.1+\left(\frac{1}{\frac{38686}{s}}+\frac{1}{29794}\right)^{-1}\right)^{-1}\right)^{-1} .
\end{aligned}
$$

The mechanical network corresponding to the admittance in (31) is shown in Fig. 14. Taking into account that a large value of damper rate is a short circuit, we remove the large damper of $c=29794 \mathrm{Ns} / \mathrm{m}$ and investigate the remaining admittance $\left(K_{1}(s)\right)$. The change in the frequency response from that of $K_{a p}(s)$ is hardly noticeable (see Fig. 13) but $J_{3}$ deteriorates by $0.6 \%$ to a value of 534.8 .

As seen in this section, it is frequently the case that an admittance obtained from the optimization algorithm may be approximated by a positive real function of the same or lower order to permit a simpler realization, while incurring only a minimal degradation in performance. This has been carried out here on a case-by-case basis without seeking a systematic method to achieve this.

\section{OPTIMIZATION OF $J_{5}$}

The generalized plant for the $J_{5}$ optimization is formed by considering $F_{s}$ as the external disturbance and $z_{s}$ as the performance output. The objective is to find a positive real $K(s)$ so that $J_{5}=\left\|T_{\hat{F}_{s} \rightarrow \hat{z}_{s}}\right\|_{\infty}$ is minimized. Here it is advantageous to allow the admittance $K(s)$ to be either proper or nonproper. The proper admittance is given by (2) and the nonproper one is given by

$$
K(s)=C_{k}\left(s I-A_{k}\right)^{-1} B_{k}+D_{k}+s E_{k}
$$

with $E_{k}>0$ in order to satisfy positive realness along with the LMI condition (3). The term $s E_{k}$ represents an inerter with inertance $E_{k}$ in parallel with the proper admittance.

\section{A. Optimization Over Proper Controllers}

From (10), the observable and controllable representation of the $J_{5}$ generalized plant is

$$
\begin{aligned}
\dot{x} & =A x+B_{1} F_{s}+B_{3} F \\
z_{s} & =C_{2} x, \quad \dot{z}_{s}-\dot{z}_{u}=C_{3} x .
\end{aligned}
$$

Given a controller $K(s)$ of order $n_{k}$, with state-space representation as in (2), let the state-space representation of the closedloop system resulting from the interconnection of the generalized plant and the controller be denoted by

$$
\left[\begin{array}{c}
\dot{x} \\
\dot{x}_{k} \\
\hline z_{s}
\end{array}\right]=\left[\begin{array}{cc}
A_{c l} & B_{c l} \\
C_{c l} & D_{c l}
\end{array}\right]\left[\begin{array}{c}
x \\
x_{k} \\
F_{s}
\end{array}\right] .
$$

Theorem 6.1: There exists a positive real controller $K(s)$ of order $n_{k}$ such that $J_{5}=\left\|T_{\hat{F}_{s} \rightarrow \hat{z}_{s}}\right\|_{\infty}<\gamma$ and $A_{c l}$ is stable, if and only if, the following problem is feasible for some $X_{c l}>0$, $X_{k}>0, \gamma>0$ and $A_{k}, B_{k}, C_{k}, D_{k}$ of compatible dimensions

$$
M<0, \quad\left[\begin{array}{cc}
A_{k}^{T} X_{k}+X_{k} A_{k} & X_{k} B_{k}-C_{k}^{T} \\
B_{k}^{T} X_{k}-C_{k} & -D_{k}^{T}-D_{k}
\end{array}\right]<0
$$

where $X_{c l}:=\left[\begin{array}{ll}X_{11} & X_{12} \\ X_{12}^{T} & X_{22}\end{array}\right]$ and $M$ is a symmetric matrix with

$$
\begin{aligned}
M_{11}= & X_{11} A+()^{T}+X_{11} B_{3} D_{k} C_{3}+()^{T} \\
& +X_{12} B_{k} C_{3}+()^{T} \\
M_{12}= & X_{11} B_{3} C_{k}+X_{12} A_{k}+A^{T} X_{12} \\
& +C_{3}^{T} D_{k}^{T} B_{3}^{T} X_{12}+C_{3}^{T} B_{k}^{T} X_{22} \\
M_{13}= & X_{11} B_{1}, M_{14}=C_{2}^{T}, M_{23}=X_{12}^{T} B_{1} \\
M_{22}= & X_{22} A_{k}+()^{T}+X_{12}^{T} B_{3} C_{k}+()^{T} \\
M_{24}= & 0_{n_{k} \times 1}, M_{34}=0, M_{33}=M_{44}=-\gamma I
\end{aligned}
$$

where ()$^{T}$ denotes the transpose of the preceding matrix. 


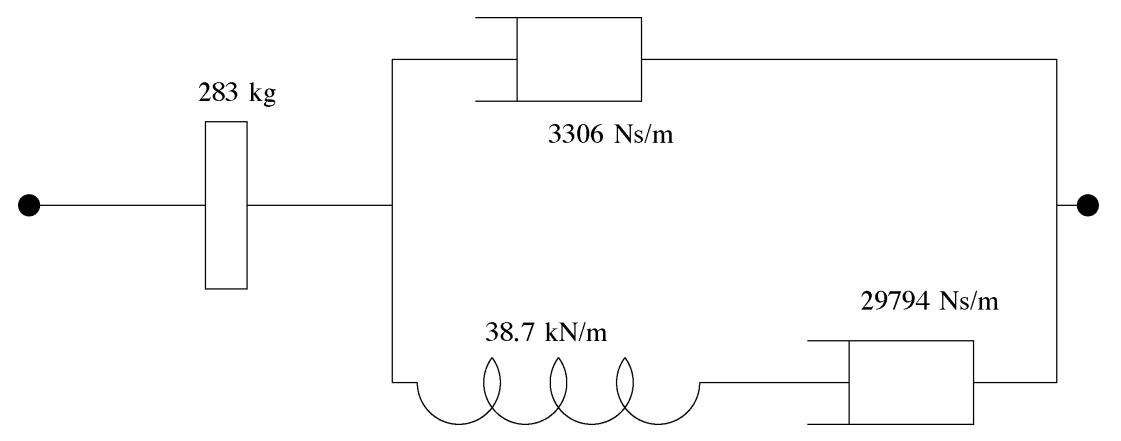

Fig. 14. Suspension layout corresponding to the admittance $K_{a p}(s)$ for $k_{s}=90 \mathrm{kN} / \mathrm{m}$.

The matrix inequality $M<0$ results by applying the Bounded Real Lemma on the closed-loop system of (35), which is a necessary and sufficient condition for the existence of a stabilizing controller that achieves an upper bound on the $\mathcal{H}_{\infty}$-norm. In the absence of the positive real constraint, the search for a stabilizing controller of order $n_{k}=n$ that minimizes the closed-loop $\mathcal{H}_{\infty}$ norm was formulated as a convex optimization problem in [15]. With the additional positive real constraint, it is not obvious how to formulate the problem as a convex problem.

The problem of Theorem 6.1 is a BMI and an iterative algorithm is proposed to solve the problem locally about a feasible starting point. The idea is to fix a subset of the decision variables so that the matrix inequality is linear with respect to the remaining decision variables and thus can be solved efficiently. The steps for the algorithm are described below.

1) Decide on the static stiffness $k_{s}$ of the suspension and on the order of the controller $n_{k}$. Give an initial controller $\left(A_{k}, B_{k}, C_{k}, D_{k}\right)$ which is positive real (as well as stabilizing).

2) For the given controller, minimize $\gamma$ over the Lyapunov matrices $X_{c l}$ and $X_{k}$ that are associated with the $\mathcal{H}_{\infty}$ bound and the positive real condition, respectively.

3) Fix $X_{c l}$ and $X_{k}$ according to the values of the previous step. Minimize $\gamma$ over the controller matrices, using as an initial starting point, the previous controller matrices and the value of $\gamma$ from the previous step.

4) Unless $\gamma$ satisfies some stopping criterion, go to step 2.

Note that there is a theoretical minimum of $J_{5}$ given by $J_{5 \min }=$ $k_{s}^{-1}+k_{t}^{-1}$ which is equal to $T_{\hat{F}_{s} \rightarrow \hat{z}_{s}}(0)$.

\section{B. Optimization Over Nonproper Controllers}

The nonproper admittance (32) can be represented in ordinary state-space form by introducing a second measurement equal to the acceleration signal $\left(\ddot{z}_{s}-\ddot{z}_{u}\right)$ as follows:

$$
\begin{gathered}
\dot{x}_{k}=A_{k} x_{k}+\left[\begin{array}{ll}
B_{k} & 0_{n_{k} \times 1}
\end{array}\right]\left[\begin{array}{l}
\dot{z}_{s}-\dot{z}_{u} \\
\ddot{z}_{s}-\ddot{z}_{u}
\end{array}\right], \quad x_{k} \in \mathbb{R}^{n_{k}} \\
F=C_{k} x_{k}+\left[\begin{array}{ll}
D_{k} & E_{k}
\end{array}\right]\left[\begin{array}{l}
\dot{z}_{s}-\dot{z}_{u} \\
\ddot{z}_{s}-\ddot{z}_{u}
\end{array}\right] .
\end{gathered}
$$

The $J_{5}$ generalized plant equations are augmented by the acceleration measurement output to give the following equation:

$$
\left[\begin{array}{c}
\dot{z}_{s}-\dot{z}_{u} \\
\ddot{z}_{s}-\ddot{z}_{u}
\end{array}\right]=\left[\begin{array}{c}
C_{3} \\
C_{4}
\end{array}\right] x+\left[\begin{array}{c}
0 \\
D_{1}
\end{array}\right] F_{s}+\left[\begin{array}{c}
0 \\
D_{2}
\end{array}\right] F
$$

where

$$
\begin{aligned}
C_{4} & =\left[\begin{array}{llll}
0 & -\frac{k_{s}}{m_{s}}-\frac{k_{s}}{m_{u}} & 0 & \frac{k_{s}}{m_{s}}+\frac{k_{s}+k_{t}}{m_{u}}
\end{array}\right] \\
D_{1} & =\frac{1}{m_{s}} \\
D_{2} & =-\frac{1}{m_{s}}-\frac{1}{m_{u}} .
\end{aligned}
$$

With the change of variable

$$
Z_{k}=\left(1-E_{k} D_{2}\right)^{-1} \Leftrightarrow E_{k}=\left(1-Z_{k}^{-1}\right) D_{2}^{-1}
$$

the closed-loop equations are given by

$$
\begin{aligned}
{\left[\begin{array}{c}
\dot{x} \\
\dot{x}_{k}
\end{array}\right]=} & {\left[\begin{array}{cc}
A+B_{3} Z_{k} D_{k} C_{3}+B_{3}\left(Z_{k}-I\right) D_{2}^{-1} C_{4} & B_{3} Z_{k} C_{k} \\
B_{k} C_{3} & A_{k}
\end{array}\right] } \\
& \times\left[\begin{array}{c}
x \\
x_{k}
\end{array}\right]+\left[\begin{array}{c}
B_{1}+B_{3}\left(Z_{k}-I\right) D_{2}^{-1} D_{1} \\
0_{n_{k} \times 1}
\end{array}\right] F_{s} \\
z_{s}= & {\left[\begin{array}{ll}
C_{2} & 0_{1 \times n_{k}}
\end{array}\right]\left[\begin{array}{c}
x \\
x_{k}
\end{array}\right] . }
\end{aligned}
$$

Theorem 6.2: There exists a nonproper, positive real admittance $K(s)$ with $x_{k} \in \mathbb{R}^{n_{k}}$ such that $J_{5}=\left\|T_{\hat{F}_{s} \rightarrow \hat{z}_{s}}\right\|_{\infty}<\gamma$ and $A_{c l}$ is stable, if and only if the following problem is feasible for some $X_{c l}>0, X_{k}>0, \gamma>0$ and $A_{k}, B_{k}, C_{k}, D_{k}, Z_{k}$ of compatible dimensions:

$$
M<0,\left[\begin{array}{cc}
A_{k}^{T} X_{k}+X_{k} A_{k} & X_{k} B_{k}-C_{k}^{T} \\
B_{k}^{T} X_{k}-C_{k} & -D_{k}^{T}-D_{k}
\end{array}\right]<0,0<Z_{k}<1
$$

where $X_{c l}:=\left[\begin{array}{ll}X_{11} & X_{12} \\ X_{12}^{T} & X_{22}\end{array}\right]$ and $M$ is a symmetric matrix with

$$
\begin{aligned}
M_{11}= & X_{11} B_{3} Z_{k} D_{k} C_{3}+()^{T}+X_{12} B_{k} C_{3}+()^{T} \\
& +X_{11} B_{3} Z_{k} D_{2}^{-1} C_{4}+()^{T}-X_{11} B_{3} D_{2}^{-1} C_{4}-()^{T} \\
& +X_{11} A+()^{T} \\
M_{12}= & X_{11} B_{3} Z_{k} C_{k}+X_{12} A_{k}+A^{T} X_{12} \\
& +C_{3}^{T} D_{k}^{T} Z_{k}^{T} B_{3}^{T} X_{12}+C_{4}^{T} D_{2}^{-T} Z_{k}^{T} B_{3}^{T} X_{12} \\
& +C_{3}^{T} B_{k}^{T} X_{22}-C_{4}^{T} D_{2}^{-T} Z_{k}^{T} B_{3}^{T} X_{12} \\
M_{13}= & X_{11} B_{1}+X_{11} B_{3} Z_{k} D_{2}^{-1} D_{1}-X_{11} B_{3} D_{2}^{-1} D_{1} \\
M_{14}= & C_{2}^{T} \quad M_{24}=0_{n_{k} \times 1} \quad M_{34}=0 \\
M_{33}= & M_{44}=-\gamma I \\
M_{22}= & X_{22} A_{k}+()^{T}+X_{12}^{T} B_{3} Z_{k} C_{k}+()^{T} \\
M_{23}= & X_{12}^{T} B_{1}+X_{12}^{T} B_{3} Z_{k} D_{2}^{-1} D_{1}-X_{12}^{T} B_{3} D_{2}^{-1} D_{1} .
\end{aligned}
$$




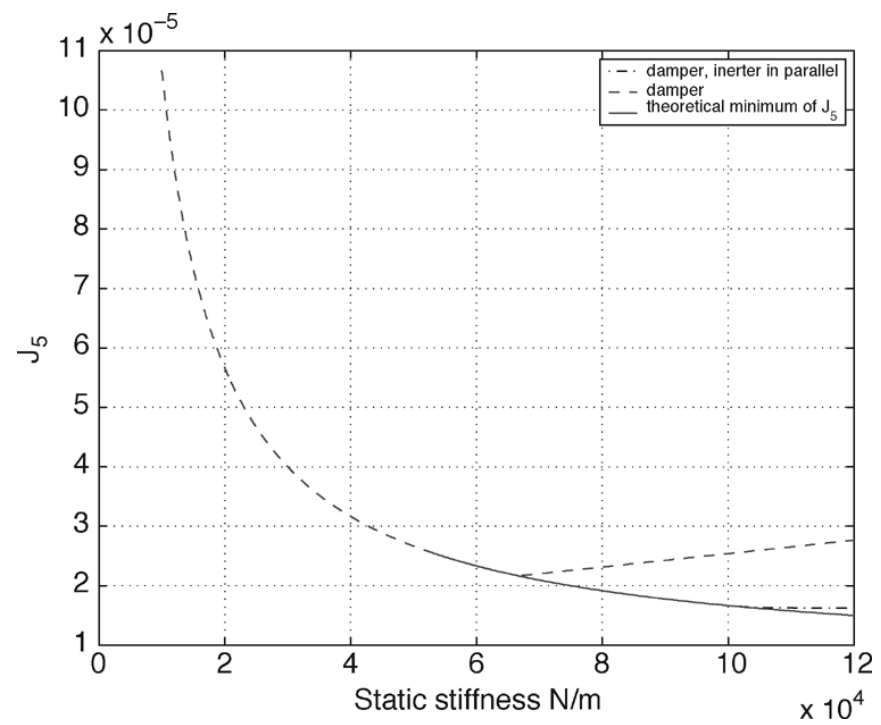

Fig. 15. Optimization results for $J_{5}$ over fixed-structure admittances.

The constraint on $Z_{k}$ ensures that $E_{k}>0$ since $D_{2}^{-1}<$ 0 . The problem in Theorem 6.2 is also solved locally using a similar iterative algorithm as the one for the proper controller case. The main difference is that the algorithm used updates the controller matrices in two steps rather than in one.

\section{C. $J_{5}$ Optimization Results}

The proposed algorithms for $J_{5}$ optimization will be assessed by comparing the obtained admittances with the fixed-structure admittances suggested in [2]. Fig. 15 presents the optimum $J_{5}$ versus static stiffness and shows the extent to which it is achieved by two fixed-structure admittances. The first is a damper, which achieves the optimum up to $k_{s}=68 \mathrm{kN} / \mathrm{m}$ and the other is a damper in parallel with an inerter that achieves the optimum up to $102 \mathrm{kN} / \mathrm{m}$.

The algorithm for proper admittances was tested at $k_{s}=$ $50 \mathrm{kN} / \mathrm{m}$. For this value of $k_{s}$ the optimum is $2.6666 \times 10^{-5}$. The algorithm produced a first-order admittance given by

$$
K(s)=6130+\left(\left(\frac{762}{s}\right)^{-1}+12591^{-1}\right)^{-1}
$$

that guarantees an upper bound of $J_{5}<2.6667 \times 10^{-5}$. The obtained admittance is more complicated than the damper proposed in [2] with optimal setting in the range $6030 \mathrm{Ns} / \mathrm{m} \leq c_{\text {opt }} \leq 19940 \mathrm{Ns} / \mathrm{m}$. An interesting observation is that if we allow the stiffness of the spring in (44) to take its extreme values $(0, \infty)$, then we recover approximately the optimal damper range.

The algorithm for the nonproper admittances was tested for $k_{s}=100 \mathrm{kN} / \mathrm{m}$. For this value of $k_{s}$ the optimum is $1.6666 \times$ $10^{-5}$. The algorithm produced a first-order admittance given by

$$
K(s)=24010 \frac{0.004}{s+0.004}+12008+292 s
$$

that guarantees an upper bound of $J_{5}<1.6667 \times 10^{-5}$. The first-order lag is relatively small so it can be neglected without causing a significant degradation in $J_{5}$. Thus, the suspension

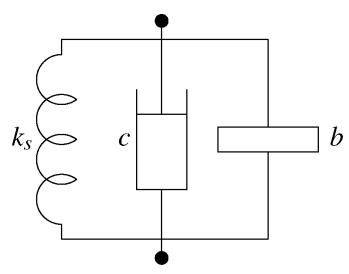

Fig. 16. Quarter-car suspension as a parallel connection of a spring, a damper, and an inerter.

consists of a parallel connection of a spring $k_{s}=100 \mathrm{kN} / \mathrm{m}$, a damper $c=12008 \mathrm{Ns} / \mathrm{m}$, and an inerter $b=292 \mathrm{~kg}$ (Fig. 16). The optimal suspension proposed in [2] for this value of $k_{s}$ is in fact a damper in parallel with an inerter with optimal values in the ranges

$$
11380 \mathrm{Ns} / \mathrm{m} \leq c_{\mathrm{opt}} \leq 12287 \mathrm{Ns} / \mathrm{m} \quad 269 \mathrm{~kg} \leq b_{\mathrm{opt}} \leq 337 \mathrm{~kg} .
$$

The suspension obtained by the LMI optimization is within the above range. Moreover the LMI algorithm managed to find a second-order suspension that achieves the theoretical minimum at the top range of $k_{s}$, which could not be achieved with the fixed-order admittances considered in [2]. The resulting admittance was given by

$$
K(s)=51450 \frac{s^{2}+14.11 s+1302}{s^{2}+67.66 s+4468}+423 s
$$

which gave a rather complicated network when using the Bott-Duffin realization method [16]. The existence of a simpler realization is currently being investigated.

\section{MULTI-OBJECTIVE OPTIMIZATION AND OTHER GENERALIZATIONS}

The practical design of vehicle suspension systems usually involves a tradeoff between a variety of performance objectives [2], [4]. It is possible to extend the techniques of the present paper to include multi-objective optimization. To illustrate this, we consider the case of the combined optimization of the measures $J_{1}$ and $J_{3}$ defined above. The approach taken here is to minimize

$$
\sqrt{(1-\lambda) \frac{J_{1}^{2}}{\hat{J}_{1}^{2}}+\lambda \frac{J_{3}^{2}}{\hat{J}_{3}^{2}}}
$$

for $0<\lambda<1$, where $\hat{J}_{1}$ and $\hat{J}_{3}$ are the optimal values obtained in the single-objective optimizations. To solve this problem, a generalized plant was formulated with the two performance outputs being the appropriate scalar multiples of $\dot{z}_{s}$ and $\int\left(z_{u}-z_{r}\right)$. A new theorem was written down which is directly analogous to Theorem 4.1 to give a necessary and sufficient condition for the achievement of a certain performance level. This characterization was again in the form of an BMI which was solved using the iterative algorithm implemented in YALMIP. This problem was solved for a range of $\lambda$ values in the interval $(0,1)$ with a fixed static stiffness $k_{s}=60 \mathrm{kN} / \mathrm{m}$. The results obtained are shown in Fig. 17.

The LMI formulation of the positive real synthesis problem allows the flexibility for further generalization. For example, the issue of model uncertainty can be included. One approach is to 


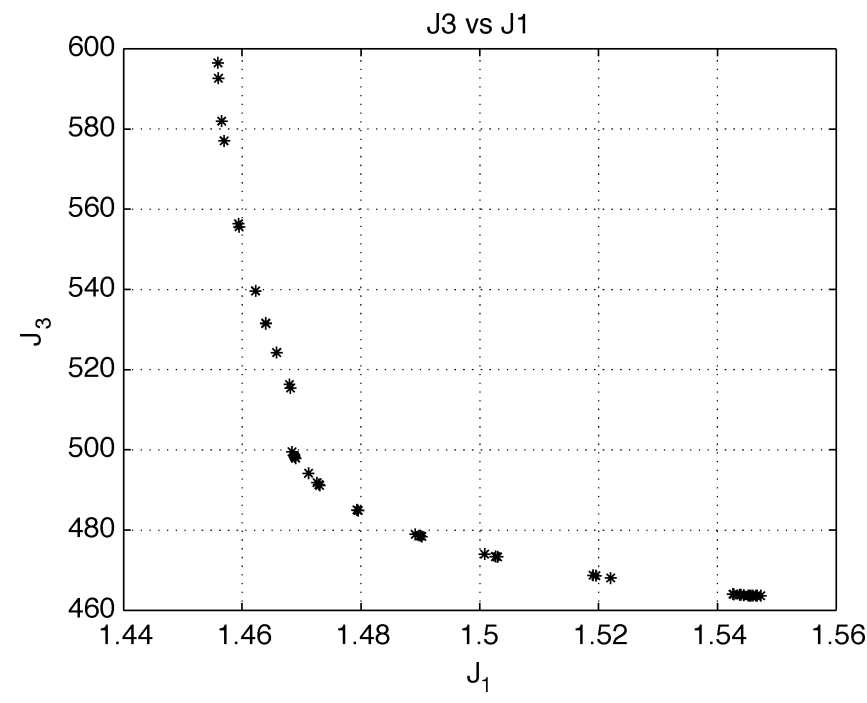

Fig. 17. Multi-objective optimization of $J_{1}$ and $J_{3}$.

use a multi-model formulation for a discrete set of parameter values. Alternatively, an LFT formulation can be used for an appropriate choice of uncertain real parameters (see [17, Lemma 3]). Optimization results were obtained for the latter approach using the uncertain parameters $m_{s}$ and $k_{t}$, but these are not included for reasons of space.

\section{EXPERIMENTAl TESTING OF A Mechanical Network INVOLVING AN INERTER}

A mechanical network comprising an inerter in series with a parallel combination of a spring and a damper was designed and built at CUED. Experimental testing of the network was carried out using the Schenck Hydraulic Test Rig shown in Fig. 18. In this setup, the displacement of the hydraulic ram can be controlled and it is possible to apply reference sinusoidal signals of different frequencies and amplitudes from a MATLAB/Simulink environment that interfaces with a DSpace processor. There also exists a load cell that measures the force through the load specimen, thus, making possible the calculation of the load admittance.

The inerter device used in the mechanical network is of a ball-screw type and was designed and built in the workshops of CUED. A picture of the device, partially disassembled, is shown in Fig. 19. The inertance of the device is achieved by the rotation of the nut to which a flywheel is attached. Inertances of 50, 130 , and $230 \mathrm{~kg}$ can be realized by using different flywheels (according to the analysis carried out on the experimental data [18]), while the actual mass of the device is about $1 \mathrm{~kg}$. A displacement sensor (LVDT) is placed across the inerter so that a calculation of the inerter admittance is possible. A high-performance damper, typical of racing car applications, is used in the network with a damper rate of $4 \mathrm{kNs} / \mathrm{m}$. The spring effect is produced through the use of a titanium spring cantilever which is supported by an L-shaped aluminium frame. The spring rate was calculated as $250 \mathrm{kN} / \mathrm{m}$. The whole load arrangement is excited at its lower end by the hydraulic ram, with the load cell located at the fixed top end of the load specimen. The particular

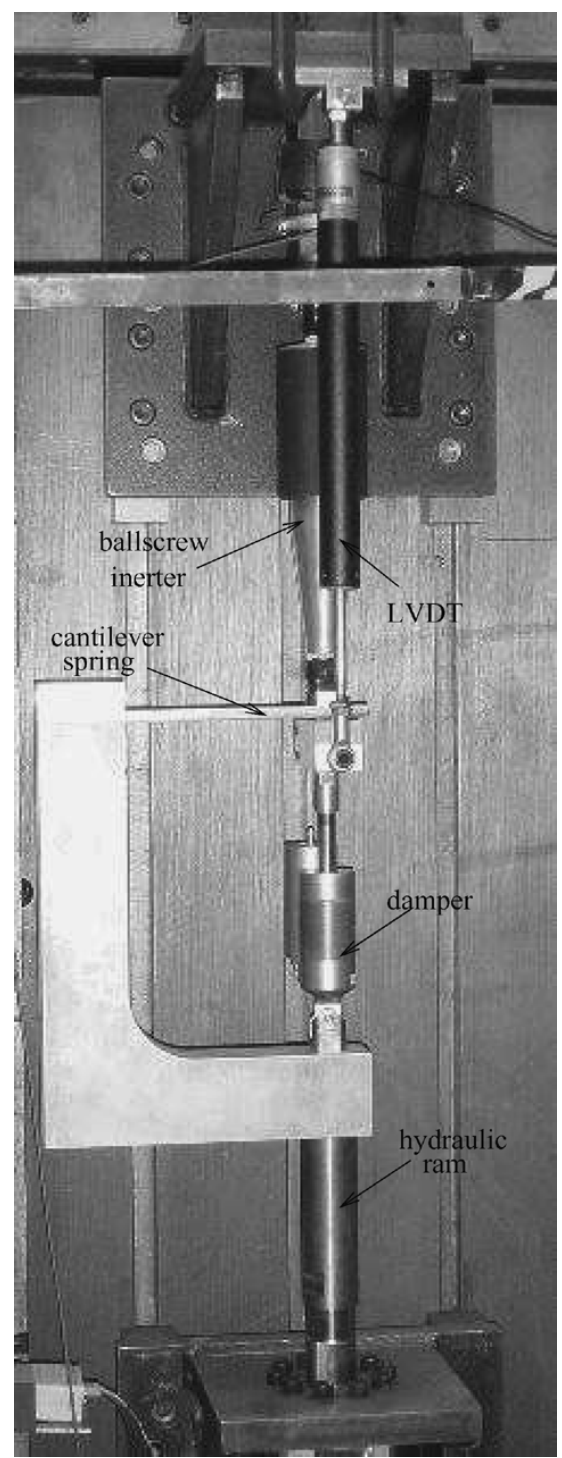

Fig. 18. Mechanical network on the hydraulic test rig.

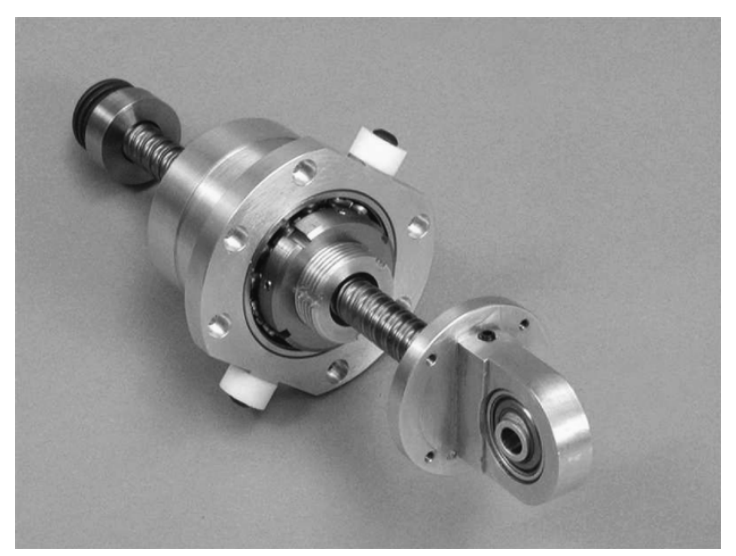

Fig. 19. Ball-screw inerter made at CUED with cover and flywheel removed.

network tested was chosen so that its admittance behaves approximately like a damper at high frequencies (see Fig. 13), and more specifically, around the crossover frequency of the rig's control system. The benefit of this choice is that no adjustment of the control system was anticipated from its normal settings 

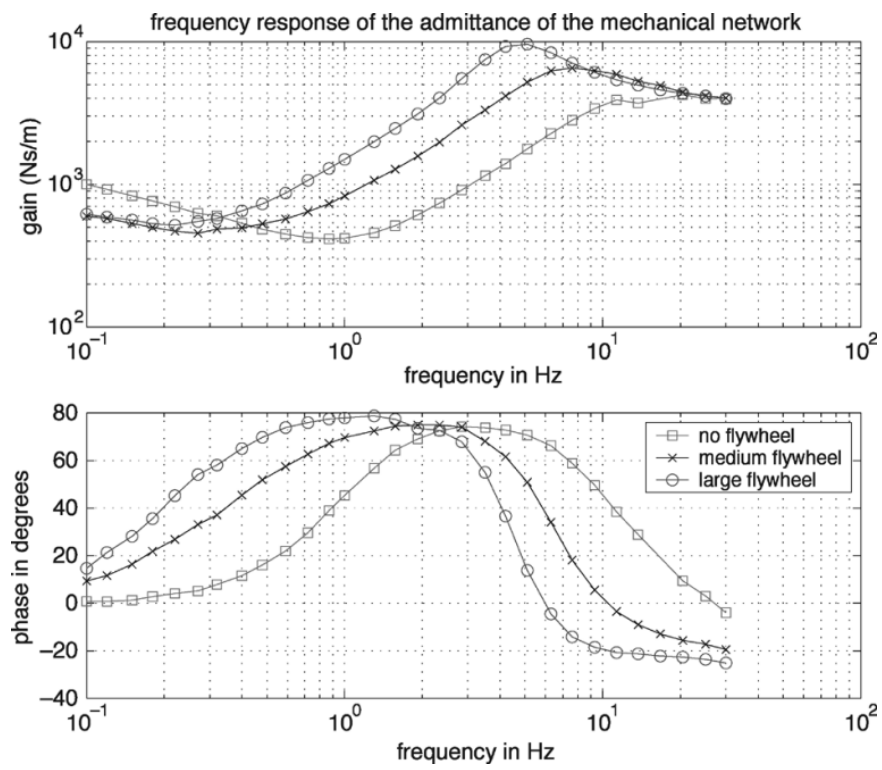

Fig. 20. Admittance of the mechanical network calculated from experimental testing.

used for testing dampers. It was known that other admittance behaviors could lead to instability, but this was not experienced in the present case.

The calculation of the admittance of both the inerter and the whole load was achieved by applying sinusoidal excitation signals in the frequency range of $0.1-30 \mathrm{~Hz}$, and recording the relevant signals which are the ram displacement, the inerter displacement, and the load force. At each frequency point, the gain and phase of the admittance of the load and the inerter were calculated using the correlation method described in [18]. The results are presented in Fig. 20 for three cases: with no flywheel in the inerter, with the medium flywheel, and with the large flywheel.

The theoretical admittance of such a network is given by

$$
Y(s)=b s\left(\frac{\frac{k}{b}}{s^{2}+\left(\frac{c}{b}\right) s+\frac{k}{b}}\right)\left(1+\left(\frac{c}{k}\right) s\right)
$$

where $b$ is the inertance, $c$ is the damper rate, and $k$ is the spring rate. The second-order system has a natural frequency of $\sqrt{k / b}$ $\mathrm{rad} / \mathrm{s}$ and a damping factor of $c /(2 \sqrt{b k})$. Furthermore, we have $\lim _{s \rightarrow \infty} Y(s)=c$ and $\lim _{s \rightarrow 0} Y(s) / s=b$, so that the theoretical network behaves like a damper at high frequencies and like an inerter at low frequencies. We see from Fig. 20 that at high frequencies the network indeed behaves like a damper of approximately $4-\mathrm{kNs} / \mathrm{m}$ and this is independent of the inertance value. In all three cases, there is an intermediate frequency range in which the experimental admittance tends toward the inerter admittance $b s$ and we get a considerable phase advance. As the flywheel inertance increases, the observed natural frequency indeed becomes smaller, and lighter damping is evident. The experimental results match the theoretical model of the admittance apart from the case when the frequency tends to zero. Interestingly, the experimental results at low frequencies are inconsistent with linear theory, since the phase tends to zero while the gain shows an increase of approximately $10 \mathrm{~dB} / \mathrm{dec}$. It is expected that the low-frequency behavior is influenced by friction in the inerter device, although, more investigation is required in order to model these effects.

\section{CONCLUSION}

The problem with synthesis of positive real controllers was formulated using matrix inequalities. Two local optimization methods were proposed to solve the bilinear matrix inequality problems in the context of suspension design for a quarter-car vehicle model. The algorithms were successful in obtaining previously found solutions when optimizing over fixed-structure admittances. In the case of the performance measure $J_{1}$, which characterizes the response of the sprung mass due to road disturbances, the proposed algorithm found alternative admittances that improve the performance measure considerably. In the case of the performance measure $J_{3}$, which characterizes the tyre normal load, the network realizations of the admittances from the optimization were shown to be relatively simple and implementable networks. In the case of the performance measure $J_{5}$, which characterizes the effect of dynamic loads on the sprung mass, the algorithm found positive real admittances which achieved a theoretical lower bound at high-static stiffness values, which previously had only been achieved at lower stiffnesses. A prototype inerter was built and tested at CUED in a mechanical network comprising one inerter, one damper, and one spring. The measured frequency responses gave a good match to the theoretical predictions over the frequency range $0.5 \mathrm{~Hz}-30 \mathrm{~Hz}$.

\section{ACKNOWLEDGMENT}

The authors are most grateful to N. Houghton, J. Beavis, B. Puddifoot, and A. Ross for their work in the design and manufacture of the inerter prototype. They would also like to thank D. Cebon for making the Vehicle Dynamics Group's hydraulic ram available to them, and $\mathrm{R}$. Roebuck for his assistance in the experiments.

\section{REFERENCES}

[1] M. C. Smith, "Synthesis of mechanical networks: The inerter," IEEE Trans. Autom. Control, vol. 47, no. 10, pp. 1648-1662, Oct. 2002.

[2] M. C. Smith and F.-C. Wang, "Performance benefits in passive vehicle suspensions employing inerters," Veh. Syst. Dynamics, vol. 42, no. 4, p. $235,2004$.

[3] S. Boyd, L. El Ghaoui, E. Feron, and B. Balakrishnan, Linear Matrix Inequalities in System and Control Theory. Philadelphia, PA: SIAM, 1994.

[4] J. Wang and D. A. Wilson, "Mixed $G L_{2} / H_{2} / G H_{2}$ control with pole placement and its application to vehicle active suspension systems," Int. J. Control, vol. 74, no. 13, pp. 1353-1369, 2001.

[5] J. C. Geromel and P. B. Gapski, "Synthesis of positive real $\mathrm{H}_{2}$ controllers," in Proc. Conf. Decision Control, Kobe, Japan, 1996, pp. 2864-2869.

[6] T. Shimomura, Y. Yamasaki, and T. Fujii, "LMI-based iterative synthesis of strictly positive real $\mathrm{H}_{2}$ controllers," in Proc. Amer. Control Conf., Chicago, IL, 2001, pp. 332-336.

[7] J. L. Shearer, A. T. Murphy, and H. H. Richardson, Introduction to System Dynamics. Reading, MA: Addison-Wesley, 1967.

[8] R. W. Newcomb, Linear Multiport Synthesis. New York: McGrawHill, 1966.

[9] O. Brune, "Synthesis of a finite two-terminal network whose drivingpoint impedance is a prescribed function of frequency," J. Math. Phys., vol. 10, pp. 191-236, 1931.

[10] R. Bott and R. J. Duffin, "Impedance synthesis without use of transformers," J. Appl. Phys., vol. 20, p. 816, 1949. 
[11] K. Zhou, C. J. Doyle, and K. Glover, Robust and Optimal Control. Englewood Cliffs, NJ: Prentice-Hall, 1996.

[12] C. Scherer, P. Gahinet, and M. Chilali, "Multi-objective output-feedback control via LMI optimization," IEEE Trans. Autom. Control, vol. 42, no. 7, pp. 896-911, Jul. 1997.

[13] A. Hassibi, J. How, and S. Boyd, "A path-following method for solving BMI problems in control," in Proc. Amer. Control Conf., San Diego, CA, 1999, pp. 1385-1389.

[14] J. Löfberg. (2004) YALMIP 3. [Online]. Available: http://control.ee.ethz.ch/joloef/yalmip.msql.

[15] P. Gahinet and P. Apkarian, "A linear matrix inequality approach to $H_{\infty}$ control," Int. J. Robust Nonlinear Control, vol. 4, pp. 421-448, 1959.

[16] E. A. Guillemin, Synthesis of Passive Networks. New York: Wiley, 1957.

[17] K. Sun and A. Packard, "Robust $H_{2}$ and $H_{\infty}$ filters for uncertain LFT systems," IEEE Trans. Autom. Control, vol. 50, no. 5, pp. 715-720, May 2005.

[18] C. Papageorgiou, "Experimental testing of inerter devices," CUED, Cambridge, U.K., Tech. Rep. CUED/F-INFENG/TR.504, Dec. 2004.

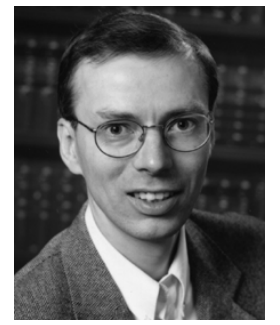

Malcolm C. Smith (M'90-SM'00-F'02) received the B.A. (M.A.) degree in mathematics in 1978 , the M.Phil. degree in control engineering and operational research in 1979, and the Ph.D. degree in control engineering in 1982, all from Cambridge University, Cambridge, U.K.

He was subsequently a Research Fellow at the German Aerospace Center, Oberpfaffenhofen, a Visiting Assistant Professor and Research Fellow with the Department of Electrical Engineering at McGill University, Montreal, QC, Canada, and an Assistant Professor with the Department of Electrical Engineering at the Ohio State University, Columbus, OH. In 1990, he joined the Engineering Department at the University of Cambridge, where he is currently a Professor. His research interests are in the areas of robust control, nonlinear systems, and automotive applications.

Dr. Smith is a co-recipient with Dr. T. T. Georgiou of the 1992 and 1999 George Axelby Best Paper Awards, in the IEEE TRANSACTIONS ON AUTOMATIC CONTROL.

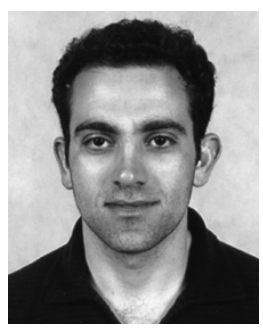

Christakis Papageorgiou received the B.A. (M.Eng.) degree in electrical and information sciences, and the Ph.D. degree in control systems from Cambridge University, Cambridge, U.K., in 1999 and 2003, respectively.

His main subject of study was the application of nonlinear control design techniques for the control of aeroservoelastic aircraft. He worked in the Control Lab as a Research Associate at the University of Cambridge, until 2004, where he carried-out research on a novel mechanical element, the inerter. Between January 2005 and January 2006, he was a Research Associate in the Department of Electrical and Computer Engineering at the University of Cyprus, Nicosia, Cyprus, working on a project in collaboration with the Engineering Department at the University of Cambridge, which was partially funded by the Research Promotion Foundation in Cyprus. His research interests include robust and optimal control with linear matrix inequalities, analysis and design of control laws for aeroservoelastic systems, vibration suppression and rejection of structural vibrations, robustness analysis of nonlinear flight control laws and synthesis, and experimental testing of passive mechanical networks for vehicle suspensions. 